Purdue University Purdue e-Pubs

ASEE IL-IN Section Conference

\title{
Designing an Escape Room Game to Develop Problem Solving and Spatial Reasoning Skills
}

Stephany Coffman-Wolph

University of Texas at Austin

Kimberlyn Gray

West Virginia University Institute of Technology

Marcia Pool

University of Illinois Urbana-Champaign

Follow this and additional works at: https:// docs.lib.purdue.edu/aseeil-insectionconference

Coffman-Wolph, Stephany; Gray, Kimberlyn; and Pool, Marcia, "Designing an Escape Room Game to Develop Problem Solving and Spatial Reasoning Skills" (2018). ASEE IL-IN Section Conference. 5.

https://docs.lib.purdue.edu/aseeil-insectionconference/2018/innov/5

This document has been made available through Purdue e-Pubs, a service of the Purdue University Libraries. Please contact epubs@purdue.edu for additional information. 


\title{
Designing an Escape Room Game to Develop Problem Solving and Spatial Reasoning Skills
}

\author{
Stephany Coffman-Wolph ${ }^{1}$, Kimberlyn Gray ${ }^{2}$, and Marcia Pool ${ }^{3}$ \\ ${ }^{1}$ University of Texas at Austin, Austin, Texas ${ }^{2}$ West Virginia University Institute \\ of Technology, Beckley, West Virginia 3University of Illinois at Urbana- \\ Champaign, Urbana, Illinois
}

\begin{abstract}
This paper describes the process of developing, piloting, and evaluating an educational and entertaining "escape room" style game for fifth grade students. The goal of this educational game is to supplement review of coursework and improve problem solving and spatial reasoning skills. The authors targeted problem solving and spatial reasoning skills because even college level students struggle with these skills, and students who have stronger abilities in these areas perform better across a variety of STEM courses. By allowing students the ability to practice and develop problem solving and spatial reasoning skills at an early age, the authors anticipate participating students will improve their confidence in utilizing these skills that are essential in scientific and technical fields [1] as well as increase their likelihood of pursuing a STEM career. Targeted surveys at pre- and post-game will be used to assess these areas from the participating students as well as their teachers' perspectives. Additionally, student performance on problems requiring employment of these skills will be measured pre- and postgame.
\end{abstract}

Keywords - K-12 Education; Educational Games; Problem Solving; Spatial Reasoning; Escape Room

Introduction, Background, and Motivation

Problem solving and spatial reasoning skills are essential to STEM careers [1], yet many students lack the practice needed to develop expertise in these areas. The authors seek to develop a mechanism through which students will be able to practice and enhance problem solving and spatial reasoning skills. The mechanism to accomplish this goal is an escape room, and the target audience is fifth grade students. Escape rooms are a popular type of game and are generally found in two varieties: (1) virtual and (2) real-life. A virtual escape room is a short app or video game where a player attempts to solve a mystery to escape the virtual environment and win the game. Virtual escape rooms generally require the user to locate hidden objects by clicking within the environment and, occasionally, combining these objects to solve a puzzle. In a real-life escape room, a group of players is "locked" in a room and work as a team to find hidden objects that are used to solve puzzles in order to "escape" the room. These real-life experiences are becoming very popular team building exercises in addition to pure entertainment experiences among groups of friends. The popularity of real-life escape rooms has created a number of new experiences, educational and/or fun, throughout the world [2] - [3]. 
The purpose of this project is to develop a real-life, easily portable escape room game containing customizable content that allows options for re-play. These two features, portability and replayability, are unique amongst typical escape room games. For purposes of educational outreach, the portability of the game is essential, and initial set-up time should be quick $(\sim 30$ minutes) with re-set for replay also being quick ( $\sim 15 \mathrm{~min})$. While the initial target group is fifth grade students, once the game has been constructed and tested, expanding on the customizability will ensure widespread outreach usability. For instance, creating content for various ages (e.g., K-12) that may be used in the customizable areas of the game will expand the population that will benefit from the game. Even with future expansion, the target audience for this game is precollege students. Therefore, the educational content in the game will be related to K-12 coursework and incorporate content from multiple subject areas such as history, English, general science, biology, chemistry, and mathematics. Additionally, to add to the customizability of the game, organizers will be able to utilize a wide variety of question types including: mathematical or calculation based, algorithm based, visually based (recognizing information from a picture), 3D spatial based, map interpretation based, logic based, or pattern recognition based. Finally, the game will require students to read content and identify relevant information thereby practicing skills needed for solving word problems.

\section{The Escape Room}

Escape Rooms are situationally themed with the participants solving some mystery. Examples of escape room themes include: archeology dig, haunted house, bank/vault heist, prison break, lab accident, sinking submarine/boat, con artist theft, steampunk, and spy interrogation; in all these roles participants play an active role (specific to the theme) to decipher the mystery. Generally, escape rooms are one-time play because there is no customizable/changeable content and the game is "spoiled" once completed.

\section{Creating the Portable Escape Room}

Although university outreach efforts often include school groups visiting the campus, most outreach efforts occur off campus via a classroom visit. Engaging students in activities that are not only educational but also fun is important. This need leads to the transportation/set-up requirements for the game and the metrics established by the authors based on their outreach experience (Table 1).

Table 1: Portable escape room requirements.

\begin{tabular}{|c|c|}
\hline Design Requirements & Established Metrics \\
\hline Easily portable & $\begin{array}{c}\text { Entire contents should } \\
\text { fit within one or two } \\
\text { easily carried plastic } \\
\text { bins }\end{array}$ \\
\hline
\end{tabular}




\begin{tabular}{|c|c|}
\hline Quick set up time & $\begin{array}{c}30 \text { minutes or less } \\
\text { (multiple people } \\
\text { assembling) }\end{array}$ \\
\hline Quick re-set for replay & $\begin{array}{c}15 \text { minutes or less } \\
\text { (multiple people } \\
\text { assembling) }\end{array}$ \\
\hline Low cost & $\$ 200$ or less \\
\hline
\end{tabular}

Spatial Reasoning and Problem Solving

Spatial reasoning is essential to engineering, architecture, graphic design, computer science, and other science fields and is fundamental for problem solving that includes visual elements [4]. Additionally, studies have shown that students with strong spatial reasoning skills have better "number sense" or understanding of relationship between quantities as these students can "see" the mathematics in their head [4]. There are many recommended activities to assist young children in building spatial reasoning skills including: (1) building blocks to create towers, stairs, and bridges, (2) tangram activities, (3) building activities using a variety of shapes and materials (toothpicks, foam shapes, marshmallows, etc.), and (4) computer programs or apps specifically designed to practice spatial reasoning skills [4]. Incorporating these spatial problems into the game (or allowing it to be a customizable option) will allow participants to practice spatial reasoning skills (multiple times with re-playability options).

\section{Example: Spatial Reasoning and Problem Solving}

The escape game will allow for the inclusion of a variety of spatial reasoning problems. The following is an example of how to incorporate a spatial reasoning problem into the game, yet in a fun way. One common element of escape room games is completing a series of tasks within a certain time limits; thus, the ability to work effectively as a team and complete activities quickly is essential to success while adding an element of excitement.

Before encountering this puzzle, students will have located several pieces of paper in a variety of shapes, but each piece of paper looks like a grouping of boxes (see Figure 1, Figure 2). The spatial reasoning in the puzzle is focused on geometric figures. Specifically, this spatial reasoning activity requires students to determine if a piece of paper with boxes (Figure 1, Figure 2) will fold into a perfect cube (without making any additional cuts to the paper) [5]. Each box on the pieces of paper will contain a letter (on both sides). The letters exposed when the perfect cube is formed will create a secret word (that will need to be deciphered in the next stage of the game). Ideally, students with stronger spatial reasoning skills will save time by studying the shapes and sorting the pieces of paper into (1) could fold into a cube and (2) not fold into a cube, based on visual analysis. Once the students have selected the best candidate to form a perfect 
cube, they will test their theory by trying to create the cube. If they are un-successful, they will continue to try until they succeed.

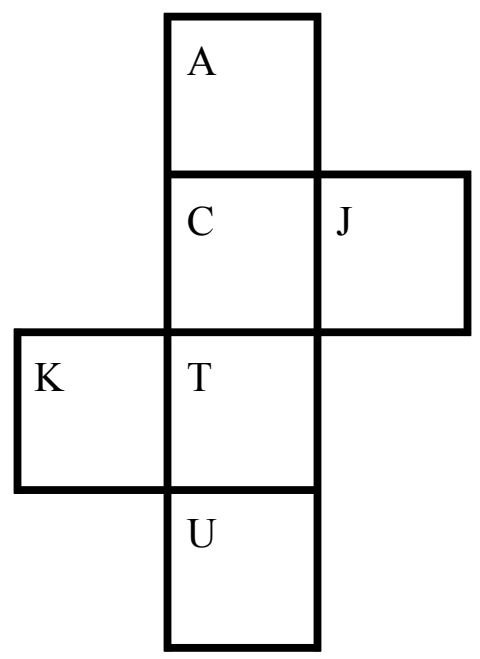

Fig. 1. Example of paper that makes a cube

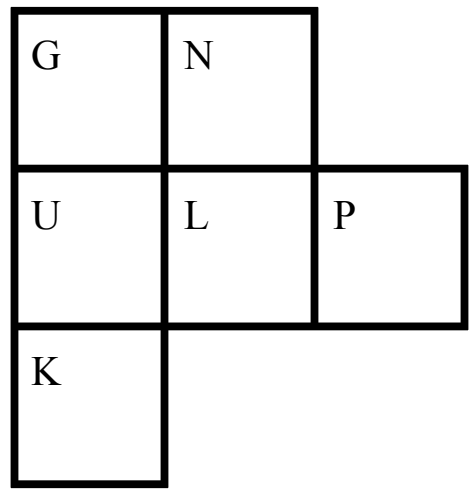

Fig. 2. Example of paper that does not make a cube

In the next stage of the game, the students will use the letters displayed on the surface of the cube (e.g., A, C, J, K, T, and U) to create a word used to decipher part of the game. Prior to this stage, the students will have found clues indicating that messages will be coded using the Caesar Cipher (a simple letter shift cipher) and will have located or calculated (e.g., averaging information obtained from another puzzle) the shift value. This experience requires students to gather and combine multiple pieces of information to decipher the letters needed to solve the puzzle.

Using the Caesar Cipher (for this example the shift value is 4) to decipher the encrypted letters leads to: E, G, N, O, X, and Y. From information in the game, students know the word is an element from the periodic table. Thus, the students will rearrange the letters (e.g., Scrabble style) to yield OXYGEN. With this puzzle, re-setting to allow re-play is easy by changing the cipher type, changing the letters on the cube, or changing the geometric shape the students try to 
assemble. Additionally, any word could be encrypted onto the cube, allowing more than the periodic table to be reviewed.

\section{Assessment Strategy}

The authors anticipate the game to be well-received and achieve the goal of increased skill practice but need to evaluate its effect in improving skills in problem solving and spatial reasoning. Therefore, to evaluate the game on (1) usability and (2) ability to improve problem solving and spatial reasoning, several methods of assessment will be employed including: preand/or post-game surveys for organizers, teachers, and students, focus groups of teachers (postgame), and evaluation of student work pre- and post-game.

\section{Pilot Program Audience}

The escape room game will be piloted in two fifth grade classes (25-30 students per class). The authors have previously worked with teacher of these classes to deliver other STEM projects (e.g., unplugged computer science activities, metric fear factor, and stream health). Problem solving and spatial reasoning skills practiced in the pilot offering will be targeted to the fifth grade level. The authors are working with the fifth grade teachers to develop appropriate problem solving activities as well as use content specific to fifth grade educational outcomes.

\section{Assessment Methods}

To assess the usability (including customizability) of the game, post-game surveys will be administered to organizers and teachers; the goal of these surveys will be to identify component of the project that are sufficient and components in which modifications/improvements are needed. To determine if there is a perceived improvement by teachers in student skills, focus groups of with the teacher(s) will be utilized; the goal of this focus group is to learn the teacher's impression of student's work post-game and correlate the teacher's impression with outcomes from student work pre- and post-game. To identify if students enjoy this type of learning activity as well as identify if their confidence in game specific content/skills improves, pre- and postgame surveys will be administered; the goal of these surveys is to learn how to improve student enjoyment and skill development. Finally, to evaluate on a quantitative level if student's skills in problem solving and spatial reasoning improved, student work associated with those skills will be evaluated pre- and post-game.

\section{Analysis of Data}

Qualitative data (survey and focus groups) will be reviewed and organized by central and minor themes. These themes will identify areas of importance that are either sufficient or need improvement. Quantitative data (review of student work pre- and post-game) will be evaluated based on modified VALUE rubrics [6], specifically aspects of the Inquiry and Analysis rubric and Problem Solving rubric. 
Future Work

After completing the pilot stage of this project and updating the game given the assessment results, the authors will begin planning for expansion beyond fifth grade. Initial expansion will remain in middle school, yet future iterations may include K-12. Having teacher advocates who are able to assist in educational level material as well as level specific outcomes is necessary; therefore, the authors will seek to engage and train teachers in this technique. After training, the teacher advocates would be able to develop their own escape room games. From the assessment perspective, once the escape room is developed, tested, and evaluated for usability and problem solving and spatial reasoning development, other instruments may be developed to explore teamwork, critical thinking, or higher levels of problem solving and spatial reasoning. Additionally, it would be beneficial to determine if any improved skills translate to activities outside the game as well as the timeline needed for improving skills through repeat play.

\section{Acknowledgment}

We thank Katy Smith, a fifth grade teacher at Ruthlawn Elementary in South Charleston, WV, for helping develop the content specific to fifth grade educational outcomes.

\section{References}

1. J. Wai et al. "Spatial Ability for STEM Domains: Aligning Over 50 Years of Cumulative Psychological Knowledge Solidifies Its Importance," Journal of Educational Psychology, vol. 101, no. 4, pp. 817-835, November 2009.

2. S. Miller, "The Art of the Escape Room", Newsweek, May, 01, 2015.

3. Z. Stone, "The Rise of Educational Escape Rooms", The Atlantic, July 28, 2016.

4. J. F. Shumway, "Building Bridges to Spatial Reasoning", Teaching Children Mathematics, vol. 20, no. 1, pp. 44-51, 2013.

5. S. Raje et al. "Connecting Spatial Reasoning Ideas in Mathematics and Chemistry", National Council of Teachers of Mathematics, vol, 107, no. 3, pp. 220-224.

6. "VALUE Rubrics" (April 2017) by Association of American Colleges \& Universities. [Online]. Available: https://www.aacu.org/value-rubrics 